\title{
Difference equations of q-Appell polynomials
}

\author{
Nazim I. Mahmudov \\ Eastern Mediterranean University, Department of Mathematics Gazimagusa, \\ TRNC, Mersin 10, Turkey \\ Email: nazim.mahmudov@emu.edu.tr
}

\begin{abstract}
In this paper, we study some properties of the $q$-Appell polynomials, including the recurrence relations and the $q$-difference equations which extend some known calssical $(q=1)$ results. We also provide the recurrence relations and the $q$-difference equations for $q$-Bernoulli polynomials, $q$-Euler polynomials, $q$-Genocchi polynomials and for newly defined $q$-Hermite polynomials, as special cases of $q$-Appell polynomials.
\end{abstract}

Key words: $q$-Appell polynomials, Lowering operators, $q$-derivative, $q$-Bernoulli polynomials, $q$-Euler polynomials, $q$-Genocchi polynomials, $q$-Hermite polynomials

\section{Introduction}

He and Ricci [4] obtained the differential equations of the Appell polynomials via the factorization method. Moreover, they found differential equations satisfied by Bernoulli and Euler polynomials as a special case. Afterward, Da-Qian Lu found differential equations for generalized Bernoulli polynomials in [6]. Recently, several interesting properties and relationships involving the classical Appell type polynomials were investigated [5]-[13].

The proof given by He and Ricci used the factorization method, which based on raising and lowering operators techniques. Note that the raising operators are not available for general polynomials, although lowering operators always exist. The proof of the main results given here for $q$-Appell polynomials does not use raising operators.

In this paper, we derive $q$-difference equations for $q$-Appell polynomials $A_{n, q}(x)$ defined in AlSalam [1]. As special cases of $q$-Appell polynomials, we also provide the $q$-difference equations for $q$-Bernoulli polynomials $B_{n, q}(x), q$-Euler polynomials $E_{n, q}(x), q$-Genocchi polynomials and for newly defined $q$-Hermite polynomials $H_{n, q}(x)$.

We briefly recall some of the properties of these polynomials. The Appell polynomials can be 
defined by considering the following generating function:

$$
A(x, t):=A_{q}(t) e_{q}(t x)=\sum_{n=0}^{\infty} A_{n, q}(x) \frac{t^{n}}{[n]_{q} !}, \quad 0<q<1,
$$

where

$$
A_{q}(t):=\sum_{n=0}^{\infty} A_{n, q} \frac{t^{n}}{[n]_{q} !}, \quad A(0) \neq 0
$$

is analytic function at $t=0$, and $A_{n, q}:=A_{n, q}(0)$, and $e_{q}(t)=\sum_{n=0}^{\infty} \frac{t^{n}}{[n]_{q} !}$.

Differentiating generation equation (1) with respect to $x$ and equating coefficients of $t^{n}$, we obtain

$$
D_{q, x} A_{n, q}(x)=[n]_{q} A_{n-1, q}(x) .
$$

Then the lowering operator $\Phi_{n}=\frac{1}{[n]_{q}} D_{q, x}$ satisfies the following operational relation:

$$
\Phi_{n} A_{n, q}(x)=A_{n-1, q}(x) .
$$

It follows that

$$
A_{n-k, q}(x)=\left(\Phi_{n-k} \circ \ldots \circ \Phi_{n}\right) A_{n, q}(x)=\frac{[n-k]_{q} !}{[n]_{q} !} D_{q, x}^{k} A_{n, q}(x) .
$$

\section{Recursion formulas and $q$-difference equations}

In this section, we derive a difference equation for the $q$-Appell polynomials $A_{n, q}(x)$ and give the recurrence relations and difference equations for the $q$-Appell polynomials.

Theorem 1 The following linear homogeneous recurrence relation for the $q$-Appell polynomials holds true:

$$
\begin{aligned}
A_{n, q}(q x) & =\frac{1}{[n]_{q}} \sum_{k=0}^{n}\left[\begin{array}{l}
n \\
k
\end{array}\right]_{q} \alpha_{n-k} q^{k} A_{k, q}(x)+x q^{n} A_{n-1, q}(x) \\
& =\frac{1}{[n]_{q}} \alpha_{0} q^{n} A_{n, q}(x)+q^{n}\left(x+\alpha_{1} q^{-1}\right) A_{n-1, q}(x)+\frac{1}{[n]_{q}} \sum_{k=0}^{n-2}\left[\begin{array}{l}
n \\
k
\end{array}\right]_{q} \alpha_{n-k} q^{k} A_{k, q}(x) .
\end{aligned}
$$

Proof. See formula (6) in the proof of Theorem 2,

Theorem 2 Assume that

$$
t \frac{D_{q, t} A_{q}(t)}{A_{q}(q t)}=\sum_{n=0}^{\infty} \alpha_{n} \frac{t^{n}}{[n]_{q} !}
$$


The q-Appell polynomials $A_{n, q}(x)$ satisfy the q-difference equation

$$
\begin{gathered}
\frac{\alpha_{n}}{[n]_{q} !} D_{q, x}^{n} A_{n, q}(x)+\frac{q \alpha_{n-1}}{[n-1]_{q} !} D_{q, x}^{n-1} A_{n, q}(x)+\ldots+\frac{q^{n-2} \alpha_{2}}{[2]_{q} !} D_{q, x}^{2} A_{n, q}(x) \\
+\frac{q^{n-1} \alpha_{1}}{[1]_{q} !} D_{q, x} A_{n, q}(x)+\frac{q^{n} \alpha_{0}}{[0]_{q} !} A_{n, q}(x)+x q^{n} D_{q, x} A_{n, q}(x)-[n]_{q} A_{n, q}(q x)=0 .
\end{gathered}
$$

Proof. Differentiating generating equation

$$
A_{q}(q x, t)=A_{q}(t) e_{q}(t q x)=\sum_{n=0}^{\infty} A_{n, q}(q x) \frac{t^{n}}{[n]_{q} !}
$$

with respect to $t$ and multiplying the obtained equality by $t$, we get the following two equations

$$
\begin{aligned}
t D_{q, t} A_{q}(q x, t) & =D_{q, t}\left(A_{q}(t) e_{q}(t q x)\right)=\left(D_{q, t} A_{q}(t)\right) e_{q}(t q x)+q x A_{q}(q t) e_{q}(t q x) \\
& =A_{q}(x, q t)\left[t \frac{D_{q, t} A_{q}(t)}{A_{q}(q t)}+t q x\right] \\
t D_{q, t} A_{q}(q x, t) & =t \sum_{n=0}^{\infty}[n]_{q} A_{n, q}(q x) \frac{t^{n-1}}{[n]_{q} !}=\sum_{n=0}^{\infty}[n]_{q} A_{n, q}(q x) \frac{t^{n}}{[n]_{q} !} .
\end{aligned}
$$

Now from the assumption (3) it follows that

$$
\begin{aligned}
\sum_{n=0}^{\infty}[n]_{q} A_{n, q}(q x) \frac{t^{n}}{[n]_{q} !} & =A_{q}(x, q t)\left[t \frac{D_{q, t} A_{q}(t)}{A_{q}(q t)}+t q x\right] \\
& =\sum_{n=0}^{\infty} q^{n} A_{n, q}(x) \frac{t^{n}}{[n]_{q} !}\left[\sum_{n=0}^{\infty} \alpha_{n} \frac{t^{n}}{[n]_{q} !}+t q x\right] \\
& =\sum_{n=0}^{\infty} \sum_{k=0}^{n}\left[\begin{array}{l}
n \\
k
\end{array}\right]_{q} \alpha_{k} q^{n-k} A_{n-k, q}(x) \frac{t^{n}}{[n]_{q} !}+x \sum_{n=0}^{\infty} q^{n+1} A_{n, q}(x) \frac{t^{n+1}}{[n]_{q} !}
\end{aligned}
$$

Equating coefficients of $t^{n}$ in equation (5), we obtain

$$
[n]_{q} A_{n, q}(q x)=\sum_{k=0}^{n}\left[\begin{array}{l}
n \\
k
\end{array}\right]_{q} \alpha_{k} q^{n-k} A_{n-k, q}(x)+x[n]_{q} q^{n} A_{n-1, q}(x) .
$$


Inserting (2) into (6) we get

$$
\begin{aligned}
{[n]_{q} A_{n, q}(q x) } & =\sum_{k=0}^{n}\left[\begin{array}{l}
n \\
k
\end{array}\right]_{q} \alpha_{k} q^{n-k} \frac{[n-k]_{q} !}{[n]_{q} !} D_{q, x}^{k} A_{n, q}(x)+x[n]_{q} q^{n} \frac{[n-1]_{q} !}{[n]_{q} !} D_{q, x} A_{n, q}(x) \\
& =\sum_{k=0}^{n} \frac{q^{n-k}}{[k]_{q} !} \alpha_{k} D_{q, x}^{k} A_{n, q}(x)+x q^{n} D_{q, x} A_{n, q}(x) \\
& =\left(\sum_{k=0}^{n} \frac{q^{n-k}}{[k]_{q} !} \alpha_{k} D_{q, x}^{k}+x q^{n} D_{q, x}\right) A_{n, q}(x) .
\end{aligned}
$$

\section{$3 \quad q$-Bernoulli polynomials}

The Bernoulli polynomials $B_{n, q}(x)$ are defined (see [2], [3]) starting from the generating function:

$$
B_{q}(x, t):=\frac{t}{e_{q}(t)-1} e_{q}(t x)=\sum_{n=0}^{\infty} B_{n, q}(x) \frac{t^{n}}{[n]_{q} !}, \quad|t|<2 \pi,
$$

and consequently, the Bernoulli numbers $b_{n, q}:=B_{n, q}(0)$ can be obtained by the generating function:

$$
B_{q}(t):=\frac{t}{e_{q}(t)-1}=\sum_{n=0}^{\infty} b_{n, q} \frac{t^{n}}{[n]_{q} !} .
$$

Theorem 3 The following linear homogeneous recurrence relation for the q-Bernoulli polynomials holds true:

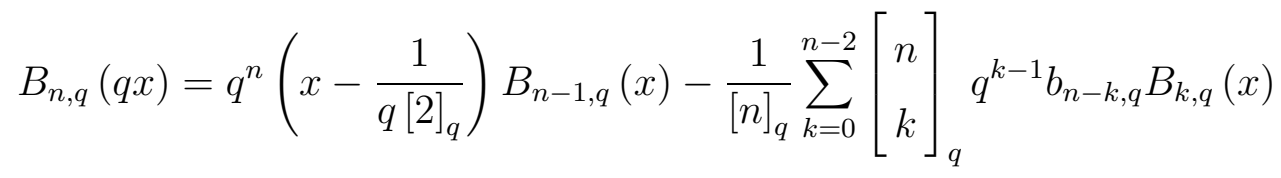

Theorem 4 The $q$-Bernoulli polynomials $B_{k, q}(x)$ satisfy the q-difference equation

$$
\begin{gathered}
\frac{b_{n, q}}{q[n]_{q} !} D_{q, x}^{n} B_{n, q}(x)+\frac{b_{n-1, q}}{[n-1]_{q} !} D_{q, x}^{n-1} B_{n, q}(x)+\ldots+q^{n-3} \frac{b_{2, q}}{[2]_{q} !} D_{q, x}^{2} B_{n, q}(x) \\
-q^{n}\left(x-\frac{1}{q[2]_{q}}\right) D_{q, x} B_{n, q}(x)+[n]_{q} B_{n, q}(q x)=0 .
\end{gathered}
$$




\section{4 q-Euler polynomials}

The Euler numbers $e_{n, q}$ can be defined by the generating function

$$
E_{q}(t):=\frac{t e_{q}(t)}{e_{q}(2 t)-1}=\sum_{n=0}^{\infty} e_{n, q} \frac{t^{n}}{[n]_{q} !} .
$$

The Euler polynomials $E_{n, q}(x)$ (see [3]) can be defined by the generating function

$$
E_{q}(x, t):=\frac{2}{e_{q}(t)+1} e_{q}(t x)=\sum_{n=0}^{\infty} E_{n, q}(x) \frac{t^{n}}{[n]_{q} !}, \quad|t|<\pi .
$$

The connection to the Euler numbers is given by

$$
e_{n, q}=2^{n} E_{n, q}\left(\frac{1}{2}\right) .
$$

Theorem 5 The following linear homogeneous recurrence relation for the q-Euler polynomials holds true:

$$
E_{n, q}(q x)=\frac{1}{2} \sum_{k=0}^{n-1}\left[\begin{array}{c}
n-1 \\
k
\end{array}\right]_{q} q^{k} E_{n-k-1} E_{k, q}(x)+x q^{n} E_{n-1, q}(x) .
$$

Theorem 6 The q-Euler polynomials $B_{k, q}(x)$ satisfy the q-difference equation

$$
\begin{gathered}
\frac{1}{2} \frac{e_{n-1, q}}{[n-1]_{q} !} D_{q, x}^{n} E_{n, q}(x)+\frac{1}{2} \frac{q e_{n-2, q}}{[n-2]_{q} !} D_{q, x}^{n-1} E_{n, q}(x)+\ldots+\frac{1}{2} \frac{q^{n-2} e_{1, q}}{[2]_{q} !} D_{q, x}^{2} E_{n, q}(x) \\
-\frac{1}{2} q^{n-1} D_{q, x} E_{n, q}(x)+x q^{n} D_{q, x} E_{n, q}(x)-[n]_{q} E_{n, q}(q x)=0 .
\end{gathered}
$$

\section{$5 \quad q$-Genocchi polynomials}

The $q$-Genocchi numbers $g_{n, q}$ can be defined by the generating function

$$
G_{q}(t):=\frac{2 t}{e_{q}(t)+1}=\sum_{n=0}^{\infty} g_{n, q} \frac{t^{n}}{[n]_{q} !} .
$$

The $q$-Genocchi polynomials $G_{n, q}(x)$ (see [3]) can be defined by the generating function

$$
G_{q}(x, t):=\frac{2 t}{e_{q}(t)+1} e_{q}(t x)=\sum_{n=0}^{\infty} G_{n, q}(x) \frac{t^{n}}{[n]_{q} !}, \quad|t|<\pi .
$$


Theorem 7 The following linear homogeneous recurrence relation for the q-Genocchi polynomials holds true:

$\frac{1}{2 q} \sum_{k=0}^{n-2}\left[\begin{array}{l}n \\ k\end{array}\right]_{q} g_{n-k, q} q^{k} G_{k, q}(x)+[n]_{q}\left(x q-\frac{1}{2 q}\right) q^{n-1} G_{n-1, q}(x)+q^{n-1} G_{n, q}(x)-[n]_{q} G_{n, q}(q x)=0$

Theorem 8 The q-Genocchi polynomials $G_{n, q}(x)$ satisfy the q-difference equation

$$
\begin{aligned}
& \frac{1}{2 q} \frac{g_{n, q}}{[n]_{q} !} D_{q, x}^{n} G_{n, q}(x)+\frac{g_{n-1, q}}{2[n-1]_{q} !} D_{q, x}^{n-1} G_{n, q}(x)+\ldots+\frac{q^{n-3} g_{2, q}}{2[2]_{q} !} D_{q, x}^{2} G_{n, q}(x) \\
& -\frac{q^{n-2}}{2} D_{q, x} G_{n, q}(x)+q^{n-1} G_{n, q}(x)+x q^{n} D_{q, x} G_{n, q}(x)-[n]_{q} G_{n, q}(q x)=0 .
\end{aligned}
$$

\section{$6 \quad q$-Hermite polynomials}

In this section we construct a $q$-Hermite polynomials and give of their some properties. Also, we derive the three-term recursive relation as well as the second-order differential equation obeyed by these new polynomials.

We define new $q$-Hermite polynomials $H_{n, q}(x)$ by means of the generating function

$$
\begin{aligned}
H_{q}(x, t) & :=H_{q}(t) e_{q}(t x)=\sum_{n=0}^{\infty} H_{n, q}(x) \frac{t^{n}}{[n]_{q} !} \\
H_{q}(t) & :=\sum_{n=0}^{\infty}(-1)^{n} q^{n(n-1)} \frac{t^{2 n}}{[2 n]_{q} ! !}, \quad[2 n]_{q} ! !=[2 n]_{q}[2 n-2]_{q} \ldots[2]_{q} .
\end{aligned}
$$

It is clear that

$$
\begin{aligned}
\lim _{q \rightarrow 1^{-}} H_{q}(x, t) & =\lim _{q \rightarrow 1^{-}} H_{q}(t) e_{q}(t x)=e^{t x} \lim _{q \rightarrow 1^{-}} \sum_{n=0}^{\infty}(-1)^{n} q^{n(n-1)} \frac{t^{2 n}}{[2 n]_{q} ! !} \\
& =e^{t x} \lim _{q \rightarrow 1^{-}} \sum_{n=0}^{\infty}(-1)^{n} \frac{t^{2 n}}{(2 n)(2 n-2) \ldots .2}=e^{t x} \lim _{q \rightarrow 1^{-}} \sum_{n=0}^{\infty}(-1)^{n} \frac{t^{2 n}}{2^{n} n !} \\
& =\exp \left(t x-\frac{t^{2}}{2}\right) .
\end{aligned}
$$

Moreover

$$
\frac{D_{q, t} H_{q}(t)}{H_{q}(q t)}=-t \quad \text { and } \quad D_{q, x} H_{n, q}(x)=[n]_{q} H_{n-1, q}(x)
$$

Theorem 9 The series form of the q-Hermite polynomial is given by

$$
H_{n, q}(x)=\sum_{k=0}^{\left[\frac{n}{2}\right]} \frac{(-1)^{k} q^{k(k-1)} x^{n-2 k}}{[2 k]_{q} ! ![n-2 k]_{q} !}
$$


Proof. Indeed, expanding the generation function $H_{n, q}(x, t)$, we have

$$
\begin{aligned}
H_{q}(x, t) & =\sum_{k=0}^{\infty}(-1)^{k} q^{k(k-1)} \frac{t^{2 k}}{[2 k]_{q} ! !} \sum_{l=0}^{\infty} x^{l} \frac{t^{l}}{[l]_{q} !} \\
& =\sum_{n=0}^{\infty} \sum_{l=0}^{\infty} \frac{(-1)^{k} q^{k(k-1)} x^{l}}{[2 k]_{q} ! ![l]_{q} !} t^{2 k+l} \quad(2 k+l=n) \\
& =\sum_{n=0}^{\infty} \sum_{k=0}^{\left[\frac{n}{2}\right]} \frac{(-1)^{k} q^{k(k-1)} x^{n-2 k}}{[2 k]_{q} ! ![n-2 k]_{q} !} t^{n}
\end{aligned}
$$

Theorem 10 The following linear homogeneous recurrence relation for the q-Hermite polynomials holds true:

$$
H_{n, q}(q x)=x q^{n} H_{n-1, q}(x)-[n-1]_{q} q^{n-2} H_{n-2, q}(x), \quad n \geq 2 .
$$

Using the recurrence relation (8), we get

$$
\begin{aligned}
& H_{0, q}(x)=1, \text { (by definition) } \\
& H_{1, q}(x)=x \\
& H_{2, q}(x)=x^{2}-1 \\
& H_{3, q}(x)=x^{3}-[3]_{q} x \\
& H_{4, q}(x)=x^{4}-\left(1+q^{2}\right)[3]_{q} x^{2}+[3]_{q} q^{2} .
\end{aligned}
$$

Theorem 11 The q-Hermite polynomials $G_{n, q}(x)$ satisfy the q-difference equation

$$
q^{n-2} D_{q, x}^{2} H_{n, q}(x)-x q^{n} D_{q, x} H_{n, q}(x)+[n]_{q} H_{n, q}(q x)=0 .
$$

In the limit when $q \rightarrow 1^{-}$, the equation (8) is reduced to the second order differential equation satisfied by the Hermite polynomials.

\section{References}

[1] W. A. Al-Salam, q-Appell polynomials. Ann. Mat. Pura Appl. (4) 771967 31-45.

[2] W. A. Al-Salam, q-Bernoulli numbers and polynomials. Math. Nachr. 171959 239-260.

[3] N. I. Mahmudov, On a class of $q$-Bernoulli and $q$-Euler polynomials. Adv. Difference Equ. 2013, 2013:108, 11 pp. 
[4] M. X. He, P. E. Ricci, Differential equation of Appell polynomials via the factorization method. J. Comput. Appl. Math. 139(2), 231-237 (2002)

[5] G. Bretti, P. E. Ricci, Multidimensional extension of the Bernoulli and Appell polynomials. Taiwan. J. Math. 8(3), 415-428 (2004).

[6] D-Q. Lu, Some properties of Bernoulli polynomials and their generalizations. Appl. Math. Lett. 24, 746-751 (2011).

[7] M.E.H. Ismail, Remarks on "Differential equation of Appell polynomials...", J. Comput. Appl. Math. 154 (2003) 243-245

[8] Y. Ben Cheikh, H. Chaggara, Connection problems via lowering operators, J. Comput. Appl. Math. 178 (2005) 45-61.

[9] M. A. Özarslan, B. Yılmaz, A set of finite order differential equations for the Appell polynomials. J. Comput. Appl. Math. 259 (2014), 108-116.

[10] B. Yılmaz, M. A. Özarslan, Differential equations for the extended 2D Bernoulli and Euler polynomials. Adv. Difference Equ. 2013, 2013:107, 16 pp.

[11] M. Garg, K. Jain, H.M. Srivastava, Some relationships between the generalized Apostol-Bernoulli polynomials and Hurwitz-Lerch zeta functions, Integral Transforms Spec. Funct. 17 (2006) 803815.

[12] Q.-M. Luo, The multiplication formulas for the Apostol-Bernoulli and Apostol-Euler polynomials of higher order, Integral Transforms Spec. Funct. 20 (2009) 377-391.

[13] H. Ozden, Y. Simsek, H.M. Srivastava, A unified presentation of the generating functions of the generalized Bernoulli, Euler and Genocchi polynomials, Comput. Math. Appl. 60 (2010) 27792787. 